\title{
THE VON NEUMANN KERNEL AND MINIMALLY ALMOST PERIODIC GROUPS
}

\author{
BY
}

\author{
SHELDON ROTHMAN
}

\begin{abstract}
We calculate the von Neumann kernel $n(G)$ of an arbitrary connected Lie group. As a consequence we see that the closed characteristic subgroup $n(G)$ is also connected. It is shown that any Levi factor of a connected Lie group is closed. Then, various characterizations of minimal almost periodicity for a connected Lie group are given. Among them is the following. A connected Lie group $G$ with radical $R$ is minimally almost periodic (m.a.p.) if and only if $G / R$ is semisimple without compact factors and $G=[G, G]^{-}$. In the special case where $R$ is also simply connected it is proven that $G=[G, G]$. This has the corollary that if the radical of a connected m.a.p. Lie group is simply connected then it is nilpotent. Next we prove that a connected m.a.p. Lie group has no nontrivial automorphisms of bounded displacement. As a consequence, if $G$ is a m.a.p. connected Lie group, $H$ is a closed subgroup of $G$ such that $G / H$ has finite volume, and $\alpha$ is an automorphism of $G$ with $\operatorname{disp}(\alpha, H)$ bounded, then $\alpha$ is trivial. Using projective limits of Lie groups we extend most of our results on the characterization of m.a.p. connected Lie groups to arbitrary locally compact connected topological groups, and finally get a new and relatively simple proof of the Freudenthal-Weil theorem.
\end{abstract}

0. Introduction. In $1934 \mathrm{~J}$. von Neumann in [21] considered the set of all elements of a topological group $G$ for which $f(x)=f(1)$ for every almost periodic function, $f$, defined on $G$. This subset, to be denoted $n(G)$, and referred to as the von Neumann kernel of $G$, was shown to be equal to the intersection of the kernels of all finite-dimensional continuous complex unitary representations of $G$; and since a unitary representation is completely reducible we need only consider irreducible representations in this intersection. Hereafter, all representations in $\S 1, \S 2$, and $\$ 4$ will be finite-dimensional continuous complex unitary representations. Since $n(G)$ is the intersection of closed normal subgroups of $G$, it is itself a closed normal subgroup of $G$. Moreover, $n(G)$ is a characteristic subgroup of $G$. For if $x \in n(G)$ and $\alpha$ is an automorphism of $G$ then for any representation, $\rho$, of $G,(\rho \circ \alpha)(x)=I$, the identity automorphism of $G$, since $\rho \circ \alpha$ is also a representation of $G$.

Von Neumann calls a topological group, $G$, minimally almost periodic, (m.a.p), if $n(G)=G$, implying $G$ has no nontrivial representations (equivalently, no irreducible representations), and maximally almost periodic, (MAP), if $n(G)=(1)$. The latter is equivalent to the statement that the representations (equivalently, the irreducible representations) separate the points. It is well known that both compact and locally compact abelian topological groups are MAP [12].

Received by the editors June 11, 1978 and, in revised form, May 30, 1979.

AMS (MOS) subject classifications (1970). Primary 22D05, 22E15; Secondary 22D10, $22 E 45$.

Key words and phrases. Von Neumann kernel, minimally almost periodic, maximally almost periodic, automorphism of bounded displacement. 
In $1936 \mathrm{H}$. Freudenthal, in [3], gave a necessary and sufficient condition for a connected topological group with countable open basis to be MAP. A. Weil's paper of 1941 [26] enables one to remove the separability restriction giving the following (Freudenthal-Weil) theorem. A locally compact connected topological group $G$ is MAP if and only if it is the direct product of a vector group and a compact group.

Since $G / n(G)$ is clearly MAP, from the Freudenthal-Weil theorem every locally compact connected group $G$ fits into a short exact sequence $(1) \rightarrow n(G) \rightarrow G \rightarrow V$ $\times P \rightarrow(1)$ where $V$ is a vector group and $P$ is a compact group.

In particular, if $G$ is an analytic group $G / n(G)$ has a faithful finite-dimensional continuous unitary representation, see e.g. [11]. As we shall see in $\S 1$, Corollary 1.8, $n(G)$ itself fits into an exact sequence

$$
(1) \rightarrow[R, R]^{-} \stackrel{i}{\rightarrow} n(G) \stackrel{\pi}{\rightarrow} V_{f}^{\perp} \cdot \pi(S) \rightarrow(1) .
$$

Various generalizations of the Freudenthal-Weil theorem for locally compact topological groups which are almost connected, i.e., $G / G_{0}$ is compact where $G_{0}$ is the identity component of $G$, are provided by M. Kuranishi [15], S. Murakami [20], and S. Grosser and M. Moskowitz [11]. Very recently, T. J. Huang in [13] gave a characterization of MAP groups in terms of transformation groups which implies several of the above-mentioned authors' results.

In $1940 \mathrm{~J}$. von Neumann and E. P. Wigner in [22] calculated $n(G)$ for various groups, and in particular showed that $S l(2, \mathbf{R})$ even discretely topologized is m.a.p. This was generalized by B. L. van der Waerden in [25] to any semisimple Lie group without compact factors. It follows for a connected semisimple Lie group $G$ that $n(G)$ is equal to the maximal compact connected normal subgroup of $G$.

Very recently in [4] H. Furstenberg gave a generalization (with a new proof) of the Borel density theorem, see e.g. [23], and its consequences to m.a.p. groups. This was further generalized by M. Moskowitz in [19]. One of the motivations for the present paper was to understand exactly the generality achieved by Furstenberg, at least in the case of connected groups.

In the present paper all groups will be assumed to be topological groups, and as mentioned previously all representations in $\S 1, \S 2$, and $\S 4$ are finite-dimensional continuous complex unitary representations and all automorphisms are bicontinuous. (However, in $\$ 3$ one considers continuous finite-dimensional but not necessarily unitary representations.) For a group $G$ and a subgroup $H$ we establish the following notation. If $G$ is locally compact and connected, the radical of $G$ is the largest closed connected solvable normal subgroup of $G$ (see K. Iwasawa, [14], for the existence of the radical).

The derived of $G$ is denoted $[G, G]$ with $[G, G]^{-}$being its closure. $Z(G)$ is the center of $G$ and $Z_{G}(H)$ is the centralizer of $H$ in $G . G_{0}$ will be the identity component of $G$, and $\hat{G}$ will denote the collection of continuous finite-dimensional complex irreducible unitary representations of $G$. We say that an automorphism, $\alpha$, of $G$, is of bounded displacement if for all $g \in G, \alpha(g) g^{-1}$ lies in some fixed compact set. In [7], F. P. Greenleaf and M. Moskowitz introduced the idea of the $H$-displacement of $\alpha$, defined to be $\operatorname{disp}(\alpha, H)=\left\{\alpha(h) h^{-1}: h \in H\right\}$. For two 
groups $A$ and $B, C=A \times{ }_{\eta} B$ denotes the semidirect product of $A$ and $B$ where $A$ is normal in $C$ and $n: B \rightarrow \operatorname{Aut}(A)$ is a homomorphism.

We now give a summary of results to be proved in this paper.

$\S 1$ is devoted to the calculation of the von Neumann kernel of an arbitrary connected Lie group. To do this it is necessary among other things to know that the Levi factor is closed. We conclude $\S 1$ with the observation that $n(G)$ is connected.

In $\$ 2$ we give various characterizations of minimal almost periodicity for a connected Lie group. The results of the previous section enable us to restrict ourselves to the case where $G$ has an abelian radical. Here, orbit considerations are the basis for the arguments. Among the characterizations is the following. A connected Lie group $G$ with radical $R$ is m.a.p. if and only if $G / R$ is semisimple without compact factors and $G=[G, G]^{-}$.

We prove in the special case where $R$ is also simply connected that $G=[G, G]$. This has the corollary that a simply connected radical of a connected m.a.p. Lie group is nilpotent.

In $\$ 3$ we prove using the techniques of $\mathrm{M}$. Moskowitz [18] together with a theorem of J. Tits [24] that a connected m.a.p. Lie group has no nontrivial automorphisms of bounded displacement. As a consequence we get a generalization of one of the results of F. P. Greenleaf, M. Moskowitz, and L. P. Rothschild [9, Corollary 5]. Namely: If $G$ is a m.a.p. connected Lie group and $H$ is a closed subgroup of $G$ such that $G / H$ has finite volume, and if $\operatorname{disp}(\alpha, H)$ is bounded then $\alpha$ is trivial. This is a generalization of Selberg's original form and gives a new proof of the corresponding result in [4]. This type of theorem will be dealt with in the case of an arbitrary connected Lie group by F. P. Greenleaf and M. Moskowitz in [7].

In $\$ 4$ making use of a decomposition due to Y. Matsushima [16], and projective limits of Lie groups, we extend most of the results of $\$ 2$ to arbitrary locally compact connected topological groups. We get as a corollary a new and relatively simple proof of the Freudenthal-Weil theorem.

Before proceeding I would like to thank my thesis advisor Professor M. Moskowitz for proposing many of the questions that are answered in the following pages and for all of his help throughout the writing of this paper.

1. Characterization of $n(G)$ for a connected group. Given a connected Lie group $G$, we let $G=R \cdot L$ be its Levi decomposition where $R$ is the radical, and $L=K S$ is the decomposition of the Levi factor into compact and noncompact parts. Let $[R, R]^{-}$denote the closure of the derived of $R$. Since $[R, R]^{-}$is a characteristic subgroup of $G$ we may form the projection $\pi: G \rightarrow G /[R, R]^{-}$. In the quotient $G /[R, R]^{-}$we have the connected abelian radical $R /[R, R]^{-}$which by wellknown structure theorems can be written as $V \times T$ where $V$ is a vector group and $T$ is a toroidal group, the $T$ being characteristic in $G /[R, R]^{-}$. To see that $T$ is actually fixed under the induced action of $\pi(L)$, (the Levi factor of $G /[R, R]^{-}$), on $R /[R, R]^{-}$, consider the continuous homomorphism from this Levi factor into the automorphism group of $T, \operatorname{Aut}(T)$, defined for $g \in \pi(L)$ by $\left.g \rightarrow \alpha_{g}\right|_{T}$. The image, $B=\left\{\left.\alpha_{g}\right|_{T}\right\}_{g \in \pi(L)}$ is a connected subgroup of the discrete group $\operatorname{Aut}(T)$ and 
therefore $B=(1)$. This implies that $\left.\alpha_{g}\right|_{T}=I$ for all $g \in \pi(L)$ and hence that $T$ is centralized by $\pi(L)$. So, $T$ is $\pi(L)$-fixed. The semisimple group $\pi(L)$ acts by conjugation on $V \times T$ and since $T$ is stable, $H$. Weyl's theorem on complete reducibility will enable us to replace $V$ by a vector group of the same dimension, also to be denoted $V$, which is $\pi(L)$-stable. To see this, let $R, \mathcal{T}$, and $\mathcal{E}$ denote the Lie algebras of $R /[R, R]^{-}, T$, and $\pi(L)$, respectively. For any representation, $\rho$, of $\pi(L)$, its differential, $d \rho$, is a linear representation of $\mathcal{L}$ such that for any subalgebra $\mathcal{H}$ of $\mathcal{L}$ with corresponding connected Lie subgroup $H$ of $\pi(L)$,

(*) $d \rho(\mathcal{E})(\mathcal{H}) \subseteq \mathcal{H}$ if and only if $\rho(\pi(\mathcal{L}))(H) \subseteq H$. Specifically, if for each $l \in \pi(\mathcal{L}), \rho(l)$ acts by conjugation on $R /[R, R]^{-}$then since $\left.\rho(\pi(L))(T) \subseteq T,{ }^{*}\right)$ implies $d \rho(\mathcal{E})(\mathcal{T}) \subseteq \mathcal{T}$. Then since $d \rho$ is a linear representation of the semisimple

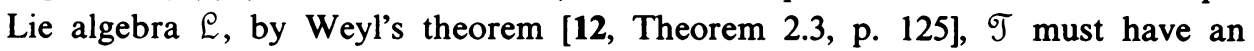
$\mathcal{L}$-stable complement in $\mathcal{R}$, written as $\mathcal{V}$. Evidently, by $(*)$, the connected subgroup of $R /[R, R]^{-}$having $\mathcal{V}$ as its Lie algebra can be chosen as our $\pi(L)$-stable $V$.

Moreover, this implies since $R /[R, R]^{-}$is abelian that $V$ is normal in $G /[R, R]^{-}$. We let $V_{f}$ denote the set of all elements of $V$ which have finite $\pi(L)$-orbit. $V_{f}$ is evidently a normal subgroup of $G /[R, R]^{-}$since it is also $\pi(L)$-stable. Arguing as above, from Weyl's theorem it must have a normal $\pi(L)$-stable complement in $G /[R, R]^{-}$, written as $V_{f}^{\perp}$. Because $\pi(L) \cdot v$, where $\cdot$ indicates the action of conjugation, is a connected set for each $v \in V$, if $v \in V_{f}$, then $\pi(L) \cdot v$ is a finite and connected set and hence must be a point. Thus, every element of $V_{f}$ is actually $\pi(L)$-fixed.

Using the notations above our first theorem is

TheOREM 1.1. If $G$ is a connected Lie group then $n(G)=\pi^{-1}\left(V_{f}^{\perp} \cdot \pi(S)\right)$.

In particular,

THEOREM 1.2. If the radical of $G$ is abelian, $n(G)=V_{f}^{\perp} \cdot S$.

For the proofs of these theorems we require the following lemma.

LEMMA 1.3. If $G$ is a connected Lie group with Levi decomposition, $G=R \cdot K S$, then both $S$ and $[R, R]^{-}$are contained in $n(G)$. If $R$ is abelian, $V_{f}^{\perp}$ is also contained in $n(G)$.

Proof of Lemma 1.3. Let $\rho$ be an irreducible representation of $G$. The restriction of $\rho$ to $S$ is a representation of $S$, and since $S$ is m.a.p. must be trivial on $S$. Thus, $S \leqslant n(G)$.

Similar to a method in [11] (see proof of Theorem 5.4), we apply A. H. Clifford's theorem [2, Theorem 1] to the normal subgroup $R$ of $G$ and,

$$
\left.\rho\right|_{R}=\nu(\chi \oplus \text { conjugates of } \chi),
$$

where $\nu$ is a natural number, and $\chi$ is an irreducible representation of $R$ (clearly, $\rho$ being continuous and unitary implies the same for $\chi$ and its conjugates). By Lie's theorem [12, Theorem 1.3, p. 119], $\chi$ and its conjugates, $\chi^{8}$, being irreducible, 
implies $\chi^{g}([R, R])=(1)$, and so by continuity $\chi^{g}\left([R, R]^{-}\right)=(1)$. Thus $\rho\left([R, R]^{-}\right)$ $=(1)$ and hence $[R, R]^{-} \subseteq n(G)$.

The standard identification of $V$ with $\hat{V}$ is given by the map $w \rightarrow \chi_{w}$, where $\chi_{w}(v)=\exp (i\langle w, v\rangle)$ (where $\langle$,$\rangle is the inner product on V$ ). If $R$ is abelian, then $V_{f}^{\perp}$ is a normal subgroup of $G$, and then by Clifford's theorem,

$$
\left.\rho\right|_{V_{f}^{\perp}}=\nu_{0}\left(\chi_{w} \oplus \text { conjugates of } \chi_{w}\right), \quad \text { for some } w \in V_{f}^{\perp},
$$

where $\nu_{0}$ is a natural number, and up to equivalence all conjugates must occur. Here, equivalence means equality since the irreducible representations of $V$ are one-dimensional, and $\chi_{w_{1}}=\chi_{w_{2}}$ if and only if $w_{1}=w_{2}$. Since the $L$-orbit of $\chi_{w}$ is infinite for all $0 \neq w \in V_{f}^{\perp}$, and $\rho$ is finite-dimensional $\left.\rho\right|_{V_{f}^{\perp}}=\nu_{0} \chi_{0}=I_{d_{\rho}}$. Thus, $V_{f}^{\perp} \subseteq n(G)$. This completes the proof of Lemma 1.3.

Proof of Theorem 1.1. We begin the proof by the reduction to the case of an abelian radical. We show that $\pi^{-1}\left(n\left(G /[R, R]^{-}\right)\right)=n(G)$. Let $\phi$ be an irreducible representation of $G$. By Theorem 1.2, $\phi\left([R, R]^{-}\right)=(1)$, and therefore $\phi$ induces a continuous representation $\tilde{\phi}$ of $G /[R, R]^{-}$, such that $\phi=\tilde{\phi} \circ \pi$. Let $y \in$ $\pi^{-1}\left(n\left(G /[R, R]^{-}\right)\right)$. Then, $\pi(y) \in n\left(G /[R, R]^{-}\right)$, and since $\phi(y)=\tilde{\phi}(\pi(y)), \phi(y)$ $=1$ and therefore $y \in n(G)$. Thus, $\pi^{-1}\left(n\left(G /[R, R]^{-}\right)\right) \subseteq n(G)$. Let $x \in n(G)$ and let $\tilde{\rho}$ be an irreducible representation of $G /[R, R]^{-}$. Then, $\tilde{\rho} \circ \pi$ is a representation of $G$, and therefore $(\tilde{\rho} \circ \pi)(x)=1$. This implies $\tilde{\rho}(\pi(x))=1$ and since $\tilde{\rho}$ was arbitrary, $\pi(x) \in n\left(G /[R, R]^{-}\right)$. Then, $x \in \pi^{-1}\left(n\left(G /[R, R]^{-}\right)\right)$, and $n(G) \subseteq$ $\pi^{-1}\left(n\left(G /[R, R]^{-}\right)\right)$. The two inclusions imply that $n(G)=\pi^{-1}\left(n\left(G /[R, R]^{-}\right)\right)$.

To complete the proof of Theorem 1.1 it remains to prove for $G=(V \times T)$. $\pi(L)$ that $n(G \eta(G))=V_{f}^{\perp} \cdot \pi(S)$. This will follow immediately from Theorem 1.2. However, we require some preliminary results.

As in [10] one says a group $G$ is a $Z$-group if $G / Z(G)$ is compact. Now by [10, Theorem 4.3] a connected group $G$ is MAP if and only if it is a $Z$-group.

THEOREM 1.4. If $G$ is a connected Lie group then any Levi factor $L$ is closed.'

Proof. Let $N_{G}(L)$ denote the normalizer of $L$ in $G$ and let $\mathcal{L}$ be its Lie algebra. First, $N_{G}(L)$ is closed. For if $x_{n} \rightarrow x$ and $x_{n} \in N_{G}(L)$ then $\operatorname{Ad} x_{n}(\mathcal{L}) \subseteq \mathcal{L}$. But Ad $x_{n} \rightarrow \operatorname{Ad} x$ and since $\mathcal{E}$ is a linear subspace of $\mathfrak{A}$, the Lie algebra of $G$, and so closed in $\mathfrak{A}$, Ad $x(\mathfrak{L}) \subseteq \mathcal{L}$. This means that $x \in N_{G}(L)$ since $L$ is an analytic group. Now as a closed subgroup of the Lie group $G, N_{G}(L)$ is also a Lie group. Hence $N_{G}(L)_{0}$, its identity component, is open, and therefore closed in $N_{G}(L)$. This means $N_{G}(L)_{0}$ is closed in $G$. But $L$ is connected and $L \subseteq N_{G}(L)$. Therefore $L \subseteq N_{G}(L)_{0} \subseteq G$. Moreover, $L$ is a maximal semisimple analytic subgroup of $N_{G}(L)_{0}$. It is one since it is a maximal semisimple analytic subgroup of $G$. If $L^{\prime}$ were a larger one in $N_{G}(L)_{0}$ it would be a larger one in $G$. Thus $L$ is a Levi factor of the closed connected Lie subgroup $N_{G}(L)_{0}$. If $L$ were closed in $N_{G}(L)_{0}$ we would be through, and since $L$ is clearly normal in $N_{G}(L)_{0}$ we may assume $L$ is normal in $G$. Now $Z(L)$ is characteristic in $L$ and therefore also normal in $G$. Since $L$ is semisimple $Z(L)$ is discrete. As a discrete normal subgroup of the connected group

\footnotetext{
${ }^{1}$ This may be well known although references are scarce.
} 
$G, Z(L)$ is central in $G$. Therefore, $\pi: G \rightarrow G / Z(L)$ is a covering map. In the connected group $G / Z(L)$ the Levi factor is clearly $L / Z(L)$. Since $L / Z(L)$ is isomorphic to the linear semisimple Lie $\operatorname{group} \operatorname{Ad}(L)$, it is closed in $G / Z(L)$ [5, Lemmas 4 and 5]. Therefore, $\pi^{-1}(L / Z(L))$ is closed by continuity. But this is just $L$.

Lemma 1.5. (a) Assume that $H$ is a closed normal subgroup of $G$ and that $H \subseteq n(G)$. If $G / H$ is $M A P$, then $H=n(G)$.

In particular,

(b) Let $G$ be a connected reductive Lie group with Levi decomposition $G=Z(G)$. $K S$. Then $n(G)=S$.

Proof. (a) By lifting representations of the maximally almost periodic group $G / H$ to $G$ we can separate all points of $G$ not in $H$. Thus, $H=n(G)$.

(b) $S$ is clearly normal in $G$, and since $S$ is closed in $K S$ [6, Theorem 1] and $K S$ is closed in $G$ (Theorem 1.4), $S$ is closed in $G$. Also, $Z K$ is closed since $K$ is compact. Therefore, $G / S \cong(Z \cdot K S) / S \cong(Z \cdot K) /(Z \cdot K \cap S)$ by the Second Isomorphism Theorem. Since $Z \cdot K$ is evidently a $Z$-group so is $Z \cdot K /(Z \cdot K \cap S)$. Thus, $G / S$ is also a $Z$-group and therefore MAP. Hence, $S=n(G)$. This completes the proof of the lemma.

Before proceeding with the proof of Theorem 1.2 we require the following

Proposition 1.6. If $G$ and $H$ are two topological groups then $n(G \times H)=n(G)$ $\times n(H)$.

Proof. Let $(g, h) \in n(G \times H)$ and let $\rho \in \hat{G}, \sigma \in \hat{H}$. Now, id $\otimes \sigma \in$ $(G \times H)^{\wedge}, p \otimes$ id $\in(G \times H)^{\wedge}$ (where id is the identity representation). So, $($ id $\otimes \sigma)(g, h)=I \otimes \sigma(h)=I$ and $(\rho \otimes \mathrm{id})(g, h)=\rho(g) \otimes I=I$. Then, since $i \otimes$ $\sigma(h)$ and $\rho(g) \otimes I$ both have one point spectrums the same must be true of $\sigma(h)$ and $\rho(g)$. But, $\sigma(h)$ and $\rho(g)$ are unitary matrices and so diagonalizable, individually. Therefore, $\sigma(h)$ and $\rho(g)$ are scalars. It now follows that $\sigma(h)=\rho(g)=I$. Then, $n(G \times H) \subseteq n(G) \times n(H)$.

For the other inclusion let $(g, h) \in n(G) \times n(H)$. Then for any $\rho \times \sigma \in$ $(G \times H)^{\wedge},(\rho \otimes \sigma)(g, h)=\rho(g) \otimes \sigma(h)=I \otimes I=I$. But every element of $(G \times H)^{\wedge}$ is the tensor product of an element of $\hat{G}$ with an element of $\hat{H}$, (see e.g. [1, Lemma 3.67]). Thus, $n(G) \times n(H) \subseteq n(G \times H)$.

Proof of Theorem 1.2. We begin the proof in the case where $G$ can be written as $(V \times T) \times{ }_{\eta} L$. Here it will be shown that $G$ is isomorphic to $T \times\left(V \times{ }_{\eta} L\right)$. Once this has been established then $n\left(T \times\left(V \times{ }_{\eta} L\right)\right)=n(T) \times n\left(V \times{ }_{\eta} L\right)=(1)$ $\times n\left(V \times{ }_{\eta} L\right)$. The first equality follows from Proposition 1.6 and the second equality holds since $T$ is compact and hence MAP. Thus, we may assume $T$ is trivial. To see the isomorphism, define the map $\phi\{((v, t), l)\}=(t,(v, l)) ; v \in V$, $t \in T, l \in L$. It is clear that $\phi$ is a homeomorphism of $(V \times T) \times{ }_{\eta} L$ onto $T \times\left(V \times{ }_{\eta} L\right)$. It is also a homomorphism in view of the fact that $V$ is $L$-stable and $T$ is $L$-fixed. Thus, $G$ is isomorphic to $T \times\left(V \times{ }_{\eta} L\right)$.

Assuming $T$ is trivial, we have $G=V \times{ }_{\eta} L$. From Lemma 1.3 both $S$ and $V_{f}^{\perp}$ 
are contained in $n(G)$ and so $V_{f}^{\perp} \times_{\eta} S$, the group generated by $V_{f}^{\perp}$ and $S$ is contained in $n(G)$. We use the notation of Lemma 1.3 for the standard identification of $V$ with $\hat{V}$. Consider the action of $G$ on $\hat{V}$.

$$
\chi^{(v, l)}\left(v^{\prime}\right)=\chi\left((v, l)\left(v^{\prime}, 1\right)(v, l)^{-1}\right)=\chi\left(n(l)\left(v^{\prime}\right)\right)
$$

where $(v, l) \in G, v^{\prime} \in V, \chi \in \hat{V}$. For $v, w \in V, l \in L$, and $\nu$ an irreducible representation of $L$, since $\chi_{w}$ acts on $V$ by scalars, $\chi_{w}(v)$ and $\nu(l)$ commute. Therefore, for each $w \in V, \chi_{w} \otimes \nu$ is a representation of $G$ if and only if for all $l \in L, v \in V$,

$$
\chi_{w}(n(l)(v))=\chi_{w}(v)
$$

From [11, Theorem 5.4] for $(v, l) \in G, w \in V, \chi_{w}^{(v, l)}=\chi\left(n(l)^{t}(w)\right)$ and therefore $\chi_{w}$ is $L$-fixed if and only if $w$ is $L$-fixed. So (1) holds if and only if $w \in V_{f}$. Clearly, for such $w$ the representation $\chi_{w} \otimes \nu$ is continuous and unitary, and irreducible because $\nu$ is already irreducible.

Let $(v, l) \in G$, where $l \in S$. We show that $(v, l) \notin n(G)$. Since $l \notin S$ and $S=n(L)\left(\right.$ Lemma 1.5(b)), there exists an irreducible representation $\nu_{l}$ of $L$ such that $\nu_{l}(l) \neq I$. But then $\left(\chi_{0} \otimes \nu_{l}\right)(v, l)=\chi_{0}(v) \nu_{l}(l)=1 \cdot \nu_{l}(l) \neq I$, and $(v, l) \notin$ $n(G)$. Consider an element $(v, l) \in G$ with $v \notin V_{f}^{\perp}$. Then, $v=v_{f}+v_{f}^{\perp}$, with $0 \neq v_{f} \in V_{f}$ and $v_{f}^{\perp} \in V^{\perp}$. For $w \in V_{f}, \chi_{w}(v)=\exp i\langle v, w\rangle$. Therefore, when $\left\langle v_{f}+v_{f}^{\perp}, w\right\rangle\left(=\left\langle v_{f}, w\right\rangle+\left\langle v_{f}^{\perp}, w\right\rangle\right) \notin \mathbf{Z}$ then $\chi_{w}(v) \neq 1$. But, $\left\langle v_{f}^{\perp}, w\right\rangle=0$ since $w \in V_{f}$. Now, for $t \in \mathbf{R},\left\langle v_{f}, t v_{f}\right\rangle=t\left\|v_{f}\right\|^{2}$, and because $t$ varies continuously, for some $t=t^{\prime}, t^{\prime}\left\|v_{f}\right\|^{2} \notin \mathbf{Z}$. Choose $w=t^{\prime} v_{f} \in V_{f}$. Then, $\chi_{w}(v) \neq 1$. Calculating, $\left(\chi_{w} \otimes \mathrm{id}\right)(v, l)=\chi_{w}(v) \cdot I \neq I$, and so $(v, l) \notin n(G)$. We have proven that $V_{f}^{\perp}$ $\times_{\eta} S \subseteq n(G)$ and that an element $(v, l) \in G-\left(V_{f}^{\perp} \times{ }_{\eta} S\right)$ is not in $n(G)$. Therefore, $n(G)=V_{f}^{\perp} \times_{\eta} S$. This completes the proof of Theorem 1.2 in the case where $G$ can be written as $R \times{ }_{\eta} L$.

For the general case of Theorem 1.2 we require:

Proposition 1.7. Assume $G$ is as in Theorem 1.2. Then the subgroups $V_{f}^{\perp} \cdot L$, $V_{f}^{\perp} \cdot T L$, and $V_{f}^{\perp} \cdot S$ are all closed in $G$.

Proof. We first prove that $V_{f}^{\perp} \cdot L$ is closed in $G$.

Case 1. $Z(L)$ is finite. Then $Z(T L)=T Z(L)$ is compact. Now $V \cap T L$ is a discrete central subgroup of $T L$ and therefore finite. But $V$ contains no finite subgroups and therefore $V \cap T L=(1)$. Since $G=V \cdot T L$ and $V$ is normal in $G$, $G=V \times{ }_{\eta} T L$. In particular as a space $G=V \times T L$. Since $V_{f}^{\perp}$ is closed in $V$ and $L$ is closed in $G$, by Theorem 1.4, and therefore in $T L, V_{f}^{\perp} \cdot L$ is closed in $G$.

Case 2. In general the semisimple group $L$ acts linearly, by conjugation on $V_{f}^{\perp}$. Since a semisimple linear group must have finite center [12, Proposition 4.1, p. 221] there exists a subgroup $\Gamma$ of finite index in $Z(L)$ which acts trivially on $V_{f}^{\perp}$. Now $V_{f}$ and $T$ are contained in $Z(G)$ and $G$ is generated by $V_{f}, V_{f}^{\perp}, T$, and $L$. Hence, $\Gamma \subseteq Z(G)$. Let $\pi: G \rightarrow G / \Gamma$ be the canonical projection. Since $Z(L)$ is discrete so is $\Gamma$, and $\pi$ is actually a covering map. Because $\Gamma \subseteq L, \pi^{-1}\left(\pi\left(V_{f}^{\perp} \cdot L\right)\right)=V_{f}^{\perp} \cdot L$ and therefore by continuity of $\pi$ it is sufficient to show $\pi\left(V_{f}^{\perp} \cdot L\right)=\pi\left(V_{f}^{\perp}\right) \cdot \pi(L)$ is closed in $G / \Gamma$. But $V_{f}$ and $V_{f}^{\perp}$ are chosen via Weyl's theorem from the Lie 
algebra $\mathfrak{A}$, and therefore they are the same in $G / \Gamma$, i.e. $\pi\left(V_{f}\right)$ is the " $V_{f}$ " of $G / \Gamma$ and $\pi\left(V_{f}^{\perp}\right)$ is the " $V_{f}^{\perp}$ " of $G / \Gamma$. Also, $\pi(\operatorname{Rad}(G))=\operatorname{Rad}(G / \Gamma)$ and therefore $\operatorname{Rad}(G / \Gamma)$ is abelian. We have $\pi(L)$ is a Levi factor of $G / \Gamma$, but $\pi(L)=(L \Gamma) / \Gamma \cong$ $L / \Gamma$ is a semisimple group with finite center. By Case $1, \pi\left(V_{f}^{\perp}\right) \cdot \pi(L)$ is closed and $V_{f}^{\perp} \cdot L$ is closed in $G$.

Now $V_{f}^{\perp} \cdot L \cdot T=V_{f}^{\perp} \cdot T L$ is also closed since $T$ is compact and $V_{f}^{\perp} \cdot L$ is closed in $G$.

Finally we prove $V_{f}^{\perp} \cdot S$ is closed. Since $V_{f}^{\perp} \cdot T \cdot L$ is closed and $V_{f}^{\perp} \cap T L=$ (1) we know $V_{f}^{\perp} \cdot T L=V_{f}^{\perp} \times{ }_{\eta} T L$. In particular as a space $V_{f}^{\perp} \cdot T L=V_{f}^{\perp} \times$ $T L$. Now $S$ is closed in $L$. Therefore, since $L$ is closed in $T L$ (in fact in $G$ ), it follows that $S$ is closed in $T L$. Hence, $V_{f}^{\perp} \cdot S$ is closed in $V_{f}^{\perp} \cdot T L$ and so also in $G$. This completes the proof of the proposition.

To complete the proof of Theorem 1.2, we construct the group $\tilde{G}=R \times{ }_{\eta} L$, where the action is conjugation in the group $G$, and define the map $\phi\{(r, l)\}=$ $(r, l)$. It is a continuous open homomorphism of $\tilde{G}$ onto $G$ mapping the subgroup $V_{f}^{\perp} \times{ }_{\eta} S$ of $\tilde{G}$ onto the subgroup $V_{f}^{\perp} \cdot S$ of $G$. Earlier in the proof we showed that $n(\tilde{G})=V_{f}^{\perp} \times{ }_{\eta} S$. Then, $V_{f}^{\perp} \times{ }_{\eta} S$ is a (closed) normal subgroup of $\tilde{G}$. Therefore, $V_{f}^{\perp} \cdot S$ is a normal subgroup of $\tilde{G}$ and moreover by Proposition 1.7 it is closed in $G$ as well. We can therefore form $G /\left(V_{f}^{\perp} \cdot S\right)$ and we let $\tilde{\phi}: \tilde{G} /\left(V_{f}^{\perp} \times{ }_{\eta} S\right) \rightarrow$ $G /\left(V_{f}^{\perp} \cdot S\right)$ be the homomorphism induced from $\phi$. The group $\tilde{G} / V_{f}^{\perp} \times{ }_{\eta} S$ is a connected MAP group, and hence a Z-group. The homomorphic image, $G /\left(V_{f}^{\perp} \cdot S\right)$, of $G /\left(V_{f}^{\perp} \times{ }_{\eta} S\right)$, under $\tilde{\phi}$, must consequently be a connected $Z$ group as well. Hence, $G /\left(V_{f}^{\perp} \cdot S\right)$ is MAP, and by Lemma 1.3 , both $S$ and $V_{f}^{\perp}$ are contained in $n(G)$. Thus, $V_{f}^{\perp} \cdot S$ is contained in $n(G)$ and so $n(G)=V_{f}^{\perp} \cdot S$ (Lemma 1.5(a)). This completes the proof of Theorem 1.2.

Proof of Theorem 1.1. We have shown that $\pi^{-1}\left(n(G) /[R, R]^{-}\right)=n(G)$. By Theorem 1.2, $n(G) /[R, R]^{-}=V_{f}^{\perp} \cdot \pi(S)$. Therefore, $n(G)=\pi^{-1}\left(V_{f}^{\perp} \cdot \pi(S)\right)$, and Theorem 1.1 is proven.

The following corollary more or less gives the structure of $n(G)$.

COROLlary 1.8. If $G$ is a connected Lie group, then $n(G)$ is a closed, characteristic, and connected subgroup of $G$. In fact one has the exact sequence

$$
(1) \rightarrow[R, R]^{-} \stackrel{i}{\rightarrow} n(G) \stackrel{\pi}{\rightarrow} V_{f}^{\perp} \cdot \pi(S) \rightarrow(1) .
$$

Proof. Since $[R, R]^{-}$is closed in $n(G)$ and $n(G) /[R, R]^{-}=V_{f}^{\perp} \cdot \pi(S)$ and $[R, R]^{-}$and $V_{f}^{\perp} \cdot \pi(S)$ are connected so is $n(G)$.

In particular, $n(G)$ has as Levi factor the noncompact part of a Levi factor of $G$. In particular, if $G$ has a compact Levi factor then $n(G)$ is solvable. In this case, the index of solvability of $n(G) \leqslant$ index of solvability of $R$.

In particular,

COROLLARY 1.9. If $G$ has abelian radical and compact Levi factor then $n(G)$ is a vector group, namely $V_{f}^{\perp}$. In this case, $\operatorname{dim} n(G) \neq 1$.

Proof. It is sufficient to show $\operatorname{dim} V_{f}^{\perp} \neq 1$. Suppose to the contrary that $\operatorname{dim} V_{f}^{\perp}=1$. Then there exists a vector $0 \neq v_{0} \in V_{f}^{\perp}$ such that $v_{0}$ is a basis of $V_{f}^{\perp}$. 
For each $k \in K, \eta(k)$ is nonsingular and hence $\eta(k)\left(v_{0}\right) \neq 0$. Therefore, there is a continuous homomorphism $\phi: K \rightarrow \mathbf{R}^{x}$ (the nonzero real numbers), such that

$$
\eta(k)\left(v_{0}\right)=\phi(k) v_{0}, \quad k \in K .
$$

So, $\phi(K)$ is a compact connected subgroup of $\mathbf{R}^{x}$, and therefore, $\phi(K)=(1)$. This implies, from (2), that $v_{0} \in V_{f}$. This is a contradiction and so $\operatorname{dim} V_{f}^{\perp} \neq 1$.

2. Minimally almost periodic groups. In this section we give various characterizations of minimally almost periodic groups, (m.a.p.), i.e., those where $n(G)=G$.

LEMMA 2.1. Let $G$ be a connected Lie group. Then $G$ is m.a.p. if and only if $G /[R, R]^{-}$is m.a.p.

Proof. Since $n(G)=\pi^{-1}\left(n\left(G /[R, R]^{-}\right)\right)$, if $G /[R, R]^{-}$is m.a.p. we have $n(G)$ $=\pi^{-1}\left(\left(G /[R, R]^{-}\right)\right)$, but the latter is $G$. Thus $G$ is m.a.p. Conversely, if $G$ is m.a.p., then so is the quotient group $G /[R, R]^{-}$.

Since Lemma 2.1 enables us to consider groups with abelian radicals we now come to:

TheOREM 2.2 Let $G$ be a connected Lie group with Levi decomposition $G=R$. $K S$. Then the following are equivalent.

(i) $G$ is m.a.p.

(ii) $G /[R, R]^{-}$can be written as $V \cdot \pi(S)$ with all nonzero elements of $V$ of unbounded $\pi(S)$-orbit.

(iii) $G / R$ has no compact factors and every nonzero element of $R /[R, R]^{-}$is of unbounded $\pi(S)$-orbit.

(iv) $G / R$ has no compact factors and $[R, R]^{-}=\left\{r \in R:[G, r] \subseteq[R, R]^{-}\right\}$.

(v) $G / R$ has no compact factors and $G=[G, G]^{-2}$.

Proof (i $\rightarrow$ ii). If $G$ is m.a.p., then $G /[R, R]^{-}$is m.a.p. and so the semisimple group $G /[R, R]^{-} / R /[R, R]^{-}$is m.a.p. and hence by Lemma 1.5 has no compact factors. Therefore, $G /[R, R]^{-}$has Levi decomposition $G /[R, R]^{-}=R /[R, R]^{-}$. $\pi(S)$ and $n\left(G /[R, R]^{-}\right)=V_{f}^{\perp} \cdot \pi(S)$ (see Theorem 1.2). Thus since $G /[R, R]^{-}$is m.a.p., $R /[R, R]^{-} \cdot \pi(S)=V_{f}^{\perp} \cdot \pi(S)$, which implies that $T=(1)$ and $R /[R, R]^{-}$ $=V_{f}^{\perp}=V$. So, $G /[R, R]^{-}=V \cdot \pi(S)$ with each nonzero element of $V\left(=V_{f}^{\perp}\right)$ of infinite $\pi(S)$-orbit. Since $\pi(S)$ is a connected semisimple Lie group without compact factors each nonzero element of $V$ has infinite $\pi(S)$-orbit if and only if it has unbounded $\pi(S)$-orbit [8, Proposition 8.4].

If (ii) holds, by Theorem 1.2, $n\left(G /[R, R]^{-}\right)=V_{f}^{\perp} \cdot \pi(S)=G /[R, R]^{-}$and so $G /[R, R]^{-}$is m.a.p. By Lemma 2.1, $G$ is m.a.p.

(i $\rightarrow$ iii). Assume that $G$ is m.a.p. Then $G / R$ is m.a.p., and since it is also semisimple must have no compact factors (Lemma 1.5(b)). That every nonzero element of $R /[R, R]^{-}$is of unbounded $\pi(S)$-orbit follows immediately from (ii).

If (iii) holds, $K=(1)$ and $T=(1)$, and hence $R /[R, R]^{-}=V$ and $G /[R, R]^{-}$ has Levi decomposition, $G /[R, R]^{-}=V \cdot \pi(S)$ with all nonzero elements of $V$ $\left(=R /[R, R]^{-}\right)$of unbounded $\pi(S)$-orbit. By (ii), $G$ is m.a.p.

\footnotetext{
${ }^{2} \mathrm{~A}$ result similar to Theorem $2.2(\mathrm{v})$ has been stated by Guivarch'.
} 
(i $\rightarrow$ iv). Suppose $G$ is m.a.p. By (iii), $G / R$ has no compact factors. Set $A=\{r \in$ $\left.R:[G, r] \subseteq[R, R]^{-}\right\}$. Clearly, $[R, R]^{-} \subseteq A$ because $[R, R]^{-}$is contained in $R$ and is a characteristic subgroup of $G$. Let $x \in A$. Then $[G, x] \subseteq[R, R]^{-}$. In particular,

$$
[S, x] \subseteq[R, R]^{-},
$$

then

$$
[\pi(S), \pi(x)]=\pi([S, x]) \subseteq \pi\left([R, R]^{-}\right)=(1) .
$$

Thus $\pi(x) \in R /[R, R]^{-}$is $\pi(s)$-fixed. By (iii), the only element of $R /[R, R]^{-}$that is $\pi(S)$-fixed is 0 . So, $\pi(x)=0$ and $x \in \operatorname{ker} \pi=[R, R]^{-}$. Therefore, $A \subseteq[R, R]^{-}$ and so equals $[R, R]^{-}$. This means

$$
[R, R]^{-}=\left\{r \in R:[S, r] \subseteq[R, R]^{-}\right\}=\left\{r \in R:[G, r] \subseteq[R, R]^{-}\right\} .
$$

Conversely, assume (iv) holds. Then $K=(1)$ and $G /[R, R]^{-}$has Levi decomposition, $G /[R, R]^{-}=R /[R, R]^{-} \cdot \pi(S)$. Suppose a nonzero element $[R, R]^{-} x \in$ $R /[R, R]^{-}$has finite (and hence trivial) $\pi(S)$-orbit and let $\cdot$ indicate the action of $\pi(S)$ on $R /[R, R]^{-}$by conjugation. Then

$$
\pi(S) \cdot \pi(x)=\pi(S) \pi(x) \pi(S)^{-1}=\pi(x) \quad \text { for all } s \in S .
$$

Thus $\pi([S, x])=(1)$ so that $[S, x] \subseteq \operatorname{ker} \pi=[R, R]^{-}$. By (5), $x \in[R, R]^{-}$. However, this is a contradiction since we assumed $[R, R]^{-} x$ to be nonzero in $R /[R, R]^{-}$. Therefore, every nonzero element of $R /[R, R]^{-}$has infinite and hence unbounded $\pi(S)$-orbit. By (iii), $G$ is m.a.p.

$(\mathrm{i} \rightarrow \mathrm{v}$ ). If (i) holds then by (iv) $G / R$ has no compact factors. If $\gamma: G \rightarrow$ $G /[G, G]^{-}$is the canonical projection then $\gamma$ is a continuous homomorphism to a locally compact abelian group. Since the latter is MAP it must have nontrivial homomorphisms into a compact group, which can be lifted to $G$. Since $G$ is m.a.p. this is impossible unless $G /[G, G]^{-}=(1)$, i.e. $G=[G, G]^{-}$.

For the reverse we may assume $G=R \cdot S$ is a Levi decomposition, with $R$ abelian. Let $\rho$ be an irreducible representation of $G$. By Clifford's theorem, the restriction $\left.\rho\right|_{R}$ can be written in the form

$$
\left.\rho\right|_{R}=\nu(\chi \oplus \text { conjugates of } \chi),
$$

where $\nu$ is a natural number, $\chi$ is an irreducible representation of $R$, and up to equivalence all conjugates of $\chi$ must occur. Since the irreducible representations of $R$ are one-dimensional, equivalence means equality. Because $\left.\rho\right|_{R}$ is finite-dimensional, $\chi$ must have a finite number of conjugates, and since $G \cdot \chi$ is connected, $\chi$ must actually be fixed under the action of $G$. Thus, $\left.\rho\right|_{R}=\nu \chi$. Therefore,

$$
\rho(r)=\nu \chi(r) I, \quad r \in R .
$$

Let $\phi: G \rightarrow \mathrm{C}$ be the continuous homomorphism defined by

$$
\phi(g)=\operatorname{det} \rho(g) \text {. }
$$

Since $C$ is abelian, $\phi\left([G, G]^{-}\right)=(1)$. However, by assumption $G[G, G]^{-}$and so $\phi(G)=(1)$. In particular, upon restricting $\phi$ to $R$ we have

$$
\phi(r)=\operatorname{det} \rho(r)=1, \quad \text { for all } r \in R .
$$


This implies that $\rho(r) \in \mathrm{SU}(n, \mathrm{C})$ for all $r \in R$. From (7), for every $r \in R, \rho(r)$ is a scalar matrix and therefore $\rho(r) \in Z(\operatorname{SU}(n, C))$. Hence $\rho(R)$ is a connected subgroup of the finite group $Z(\mathrm{SU}(n, \mathrm{C}))$, and so $\rho(R)=I$.

Consider the following commutative diagram:

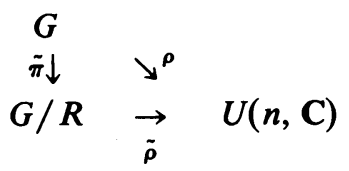

where $\tilde{\pi}$ is the canonical projection of $G$ onto $G / R$ and $\tilde{\rho}$ is the representation of $G / R$ induced from $\rho$. Evidently, $\tilde{\rho}$ must be trivial since $G / R$ is semisimple without compact factors and so m.a.p. Therefore, $\rho$ must be trivial and since $\rho$ was arbitrary, $G$ is m.a.p. This completes the proof of Theorem 2.2.

In the case of simply connected radical we can say somewhat more.

TheOREM 2.3. Assume $G$ is a connected Lie group with Levi decomposition, $G=R \cdot K S$.

(a) If $G$ is m.a.p. and $R$ is simply connected, then $G=[G, G]$.

In particular,

(b) If $G$ is m.a.p. and $R$ is abelian or $G$ is simply connected, then $G=[G, G]$.

Proof. We will first prove the result for the special case where $R$ is a vector group $V$. By Theorem 2.2(ii),

$G=V \cdot S$ with all nonzero elements of $V$ of unbounded $S$-orbit.

Calculating for $v \in V, s \in S$,

$$
[v, s]=[(v, 1),(1, s)]=(s \cdot v-v, 1),
$$

where - indicates the action of $S$ on $V$ by conjugation. Put $H=$ linear span $\{s \cdot v-v: s \in S, v \in V\} . H$ can be seen to be an $S$-submodule of $V$ as follows. For $s, t \in S, v \in V$,

$$
\begin{aligned}
t \cdot(s \cdot v-v) & =t \cdot(s \cdot v)-t \cdot v \\
& =t \cdot(s \cdot v)-s \cdot v+s \cdot v-v+v-t \cdot v \\
& =[t \cdot(s \cdot v)-s \cdot v]+[s \cdot v-v]-[t \cdot v-v] .
\end{aligned}
$$

All expressions in brackets in the last line above are in $H$ so $t \cdot(s \cdot v-v)$ is also in $H$. This shows that $H$ is an $S$-submodule of $V$. Observe that $H \neq(0)$ if $V \neq(0)$ since $S$ acts on nonzero elements of $V$ by unbounded orbits. Suppose $V$ is irreducible. By (11) the linear $\operatorname{span}\{[v, s]: s \in S, v \in V\}=$ linear $\operatorname{span}\{s \cdot v-v$ : $s \in S, v \in V\}$, and so,

$$
[V, S]=\text { linear } \operatorname{span}\{s \cdot v-v: s \in S, v \in V\}=H .
$$

Thus, if $V$ is irreducible, $[V, S]=H=V$.

In the case where $R=V$ is not necessarily irreducible, since $S$ is semisimple Weyl's theorem enables us to write $V=W_{1} \oplus \cdots \oplus W_{k}$, where each $W_{i}$ is an irreducible $S$-submodule of $V$. We have shown above that for each $i, H_{i}=$ linear $\operatorname{span}\left\{s \cdot w_{i}-w_{i}: s \in S, v \in V\right\}$ is an $S$-submodule, respectively, of $W_{i}$, with $H_{i} \neq(0)$ if $W_{i} \neq(0)$ since $S$ acts by unbounded orbits on all nonzero elements of 
$W_{i}$. As above, for each $i, H_{i}=W_{i}=\left[W_{i}, S\right]$, and hence

$$
[V, S]=\left[W_{1} \oplus \cdots \oplus W_{k}, S\right]=\sum_{i=1}^{k}\left[W_{i}, S\right]=\sum_{i=1}^{k} W_{i}=V .
$$

We know $[S, S]=S$ is semisimple. From this fact and (13), we see that $[G, G]$ contains both $V$ and $S$, and so must contain $V \cdot S$, the group generated by $V$ and $S$. Since $V \cdot S=G$, by $(10)$, we conclude that $[G, G]$ contains $G$ and so $G=$ $[G, G]$.

In the general case, Theorem 2.2(ii) implies that $R /[R, R]^{-}$is a vector group. By Lemma $2.1, G /[R, R]^{-}$is m.a.p. and so by the special case proven above for a m.a.p. group whose radical is a vector group, $G /[R, R]^{-}=\left[G /[R, R]^{-}\right.$, $\left.G /[R, R]^{-}\right]$. This implies $\pi([G, G])=[\pi(G), \pi(G)]=\pi(G)$. Therefore,

$$
\operatorname{ker} \pi \cdot[G, G]=G \text {. }
$$

But $\operatorname{ker} \pi=[R, R]^{-}$and $[R, R]^{-}=[R, R]$ since $R$ is simply connected [12, Theorem 1.2, p. 135]. Thus ker $\pi=[R, R] \subseteq[G, G]$ and (14) becomes $[G, G]=G$. This completes the proof of Theorem 2.3(a).

To prove (b) we note that if $G$ is simply connected, then $R$ is simply connected and so $G=[G, G]$ by (a). The proof of Theorem 2.2 shows that if $G$ is m.a.p. and $R$ is abelian then it contains no nontrivial torus and hence is simply connected. Then, $G=[G, G]$ follows again by (a).

COROLlARY 2.4. If $G$ is a connected m.a.p. Lie group with simply connected radical $R$, then $R$ is nilpotent.

Proof. By Theorem 2.3, $G=[G, G]$, and therefore $R=R \cap G=R \cap[G, G]$. $R \cap[G, G]$ is nilpotent because it is a connected Lie group and its Lie algebra, $\delta=\Re \cap[\mathcal{G}, \mathcal{G}]$ is nilpotent [12, Theorem 3.2, p. 128]. Hence, $R$ is nilpotent.

We close by constructing an example of a connected m.a.p. Lie group $G$ where $[G, G] \neq G$. This will show that we may not drop the assumption that the radical of $G$ is simply connected in Theorem 2.3 .

EXAMPLE 2.5. Let $H=\{(x, y, z, t): x, y, z, t \in \mathbf{R}\}$ with the manifold structure of $\mathbf{R}^{4}$ and with its multiplication defined by

$$
\begin{aligned}
\left(x_{1}, y_{1}, z_{1}, t_{1}\right) & \left(x_{2}, y_{2}, z_{2}, t_{2}\right) \\
& =\left(x_{1}+x_{2}+z_{1} t_{2}, y_{1}+y_{2}+\alpha z_{1} t_{2}, z_{1}+z_{2}, t_{1}+t_{2}\right),
\end{aligned}
$$

where $\alpha$ is a fixed irrational real number. Let $D$ be the discrete central subgroup of $H$ consisting of the elements $(p, q, 0,0)$ with arbitrary integers $p$ and $q$. Form $H / D$. We observe that $H / D$ is not simply connected [12, Example 2, p. 140]. Let $l=D\left(x_{1}, y_{1}, z_{1}, t_{1}\right)$ and $m=D\left(x_{2}, y_{2}, z_{2}, t_{2}\right)$ be elements of $H / D$. We have

$$
l^{-1}=D\left(-x_{1}+z_{1} t_{1},-y_{1}+\alpha z_{1} t_{1},-z_{1},-t_{1}\right)
$$

and

$$
m^{-1}=D\left(-x_{2}+z_{2} t_{2},-y_{2}+\alpha z_{2} t_{2},-z_{2},-t_{2}\right) \text {. }
$$


Thus, using (15),

$$
\begin{aligned}
{[l, m]=} & l m l^{-1} m^{-1} \\
= & D\left(x_{1}, y_{1}, z_{1}, t_{1}\right) D\left(x_{2}, y_{2}, z_{2}, t_{2}\right) D\left(-x_{1}+z_{1} t_{1}, y_{1}+\alpha z_{1} t_{1},-z_{1},-t_{1}\right) \\
& \cdot D\left(-x_{2}+z_{2} t_{2},-y_{2}+\alpha z_{2} t_{2},-z_{2},-t_{2}\right) \\
= & D\left(x_{1}+x_{2}+z_{1} t_{2}, y_{1}+y_{2}+\alpha z_{1} t_{2}, z_{1}+z_{2}, t_{1}+t_{2}\right) \\
& \cdot D\left(-x_{1}-x_{2}+z_{1} t_{1}+z_{2} t_{2}+z_{1} t_{2},-y_{1}-y_{2}\right. \\
& \left.\quad+\alpha\left(z_{1} t_{1}+z_{2} t_{2}+z_{1} t_{2}\right),-z_{1}-z_{2},-t_{1}-t_{2}\right) \\
= & D\left(z_{1} t_{2}+z_{1} t_{1}+z_{1} t_{2}+\left(z_{1}+z_{2}\right)\left(-t_{1}-t_{2}\right),\right. \\
& \left.\alpha\left[z_{1} t_{2}+z_{1} t_{1}+z_{1} t_{2}+\left(z_{1}+z_{2}\right)\left(-t_{1}-t_{2}\right)\right], 0,0\right) .
\end{aligned}
$$

Simplifying we get

$$
[l, m]=D\left(z_{1} t_{2}-z_{2} t_{1}, \alpha\left(z_{1} t_{2}-z_{2} t_{1}\right), 0,0\right) .
$$

Then, $[H / D, H / D]=$ group generated $\{[l, m] \in H / D\}$, and since $\alpha$ is irrational (16) implies that

$$
[H / D, H / D]^{-}=\{D(x, y, 0,0): x, y \in \mathbf{R}\} .
$$

Consider the action of $\operatorname{Sl}(2, \mathbf{R})$ on $H$ defined for

$$
s=\left[\begin{array}{ll}
s_{11} & s_{12} \\
s_{21} & s_{22}
\end{array}\right] \in S l(2, \mathbf{R}),
$$

and $(x, y, z, t) \in H$ by

$$
s \cdot(x, y, z, t)=\left(x, y, s_{11} z+s_{12} t, s_{21} z+s_{22} t\right) .
$$

We have

$$
s \cdot(p, q, 0,0)=(p, q, 0,0) \text { for all } p, q, \in \mathbf{R}, \quad s \in S l(2, \mathbf{R}) .
$$

In particular, (15) holds for all $p, q, \in \mathbf{Z}, s \in S l(2, \mathbf{R})$. Thus, $S l(2, \mathbf{R})$ acts trivially on $D$ and we have an induced action of $\operatorname{Sl}(2, \mathbf{R})$ on $H / D$ defined by

$$
s \cdot D h=D(s \cdot h), \quad \text { for } s \in S l(2, \mathbf{R}), \quad D h \in H / D,
$$

where $s \cdot h$ is the action of $S l(2, \mathbf{R})$ on $H$ defined by (18). Using (20) define $G=H / D \cdot S l(2, \mathbf{R})$,

Claim. (i) $G$ is a connected m.a.p. Lie group.

(ii) $[G, G] \neq G$.

PRoof. (i) It is clear that $G$ is a connected Lie group. The radical of $G$ is $H / D$ and $G /(H / D)$ has no compact factors since $S l(2, \mathbf{R})$ has none. Since $[H / D, H / D]^{-}$is a characteristic subgroup of $G$ we can consider the induced action of $S l(2, \mathbf{R})$ on $(H / D) /[H / D, H / D]^{-}$. For $a=[H / D, H / D]^{-} D(x, y, z, t)$ $\in(H / D) /[H, D, H / D]^{-}$and

$$
\begin{gathered}
s=\left[\begin{array}{ll}
s_{11} & s_{12} \\
s_{21} & s_{22}
\end{array}\right] \in S l(2, \mathbf{R}), \\
s \cdot a=[H / D, H / D] D\left(x, y, s_{11} z+s_{12} t, s_{21} z+s_{22} t\right) .
\end{gathered}
$$


Observe that the usual action of $\operatorname{Sl}(2, \mathbf{R})$ on $\mathbf{R}^{2}$,

$$
\left[\begin{array}{ll}
s_{11} & s_{12} \\
s_{21} & s_{22}
\end{array}\right]\left[\begin{array}{l}
z \\
t
\end{array}\right]=\left(s_{11} z+s_{12} t, s_{21} z+s_{22} t\right)
$$

is such that every nonzero element $\left[\begin{array}{l}z \\ t\end{array}\right] \in \mathbf{R}^{2}$ has an infinite, and hence unbounded $S l(2, \mathbf{R})$-orbit. Then, from (21), the element $a$ above with $z$ and $t$ not both zero has unbounded $S l(2, \mathbf{R})$-orbit. Such elements, $a$, are the nonzero elements of $(H / D) /[H / D, H / D]^{-}$(see (17)), and so we have shown that $S l(2, \mathbf{R})$ acts by unbounded orbits on every nonzero element of $(H / D) /[H / D, H / D]^{-}$. Hence, $G$ is m.a.p. (Theorem 2.2(iii)).

(ii) We calculate $[G, G]$. Let $a=D\left(\left(x_{1}, y_{1}, z_{1}, t_{1}\right), s\right)$ and $b=D\left(\left(x_{2}, y_{2}, z_{2}, t_{2}\right), p\right)$ be elements of $G$ where

$$
s=\left[\begin{array}{ll}
s_{11} & s_{12} \\
s_{21} & s_{22}
\end{array}\right] \text { and } p=\left[\begin{array}{ll}
p_{11} & p_{12} \\
p_{21} & p_{22}
\end{array}\right]
$$

We have

$$
\begin{aligned}
a^{-1}=D\left(\left(-x_{1}-z_{1}[\right.\right. & \left.s_{21}\left(s_{12} t_{1}-s_{22} z_{1}\right)+s_{22}\left(s_{21} z_{1}-s_{11} t_{1}\right)\right] \\
-y_{1}-\alpha\left\{z_{1}\left[s_{21}\left(s_{12} t_{1}-s_{22} z_{1}\right)+s_{22}\left(s_{21} z_{1}-s_{11} t_{1}\right)\right]\right\} & \left.\left.s_{12} t_{1}-s_{22} z_{1}, s_{21} z_{1}-s_{11} t_{1}\right), s^{-1}\right)
\end{aligned}
$$

and

$$
\begin{aligned}
b^{-1}=D\left(\left(-x_{2}-z_{2}\left[p_{21}\left(p_{12} t_{2}-p_{22} z_{2}\right)+p_{22}\left(p_{21} z_{2}-p_{11} t_{2}\right)\right]\right.\right. \\
-y_{2}-\alpha\left\{z_{2}\left[p_{21}\left(p_{12} t_{2}-p_{22} z_{2}\right)+p_{22}\left(p_{21} z_{2}-p_{11} t_{2}\right)\right]\right\} \\
\left.\left.p_{12} t_{2}-p_{22} z_{2}, p_{21} z_{2}-p_{11} t_{2}\right), p^{-1}\right) .
\end{aligned}
$$

We have

$$
a b=D\left(\left(x_{1}+x_{2}+z_{1} c, y_{1}+y_{2}+a z_{1} c,{ }^{*}, * *\right), s p\right)
$$

and

$$
a^{-1} b^{-1}=D\left(\left(-x_{1}-x_{2}-d,-y_{1}-y_{2}-\alpha d,{ }^{* \prime},\left({ }^{* *}\right)^{\prime}\right), s^{-1} p^{-1}\right)
$$

and

$$
\begin{aligned}
{[a, b]=} & a b a^{-1} b^{-1}=D\left(\left(x_{1}+x_{2}+z_{1} c, y_{1}+y_{2}+\alpha z_{1} c,{ }^{*},{ }^{* *}\right), s p\right) \\
& \cdot D\left(\left(-x_{1}-x_{2}-d,-y_{1}-y_{2}-\alpha d,{ }^{* \prime} \cdot\left({ }^{* *}\right)^{\prime}\right), s^{-1} p^{-1}\right)
\end{aligned}
$$

where $c=s_{21} z_{2}+s_{22} t_{2}$, and

$$
\begin{aligned}
d= & z_{1}\left[s_{21}\left(s_{12} t_{1}-s_{22} z_{1}\right)+s_{22}\left(s_{1} z_{1}-s_{11} t_{1}\right)\right] \\
& +z_{2}\left[p_{21}\left(p_{12} t_{2}-p_{22} z_{2}\right)+p_{22}\left(p_{21} z_{2}-p_{11} t_{2}\right)\right] \\
& +\left[s_{12} t_{1}-s_{22} z_{1}\right]\left[s_{21}\left(p_{12} t_{2}-p_{22} z_{2}\right)+s_{22}\left(p_{21} z_{2}-p_{11} t_{2}\right)\right]
\end{aligned}
$$

Continuing,

$$
\begin{aligned}
& {[a, b]=D\left(\left(z_{1} c-d+\left[{ }^{*}\right]\left(s_{21}\left({ }^{* \prime}\right)+s_{22}\left({ }^{* *}\right)^{\prime}\right),\right.\right.} \\
& \left.\left.\alpha\left(z_{1} c-d+\left[{ }^{*}\right]\left(s_{21}\left({ }^{* \prime}\right)+s_{22}\left({ }^{* *}\right)^{\prime}\right),{ }^{* \prime \prime},\left({ }^{* *}\right)^{\prime \prime}\right)\right), s p s^{-1} p^{-1}\right) .
\end{aligned}
$$


Letting $\left.\varepsilon=z_{1} c-d+\left[{ }^{*}\right]\left(s_{21}{ }^{* \prime}\right)+s_{22}\left({ }^{* *}\right)^{\prime}\right)$, we get

$$
[a, b]=D\left(\left(\varepsilon, \alpha \varepsilon,^{* \prime \prime},\left({ }^{* *}\right)^{\prime \prime}\right), s p s^{-1} p^{-1}\right) \text {. }
$$

From (22), we see that any product of elements $[a, b]$ of the form in (24) will still have that form. Thus $[G, G]=\operatorname{group}$ generated $\{[a, b]: a, b \in G\}$ cannot be equal to all of $G$. This completes the proof of the claim.

3. Automorphisms of bounded displacement and homogeneous spaces of finite volume for minimally almost periodic groups. Recall, as in Tits [24], that an automorphism, $\alpha$, of $G$, has bounded displacement if for all $g \in G, \alpha(g) g^{-1}$ lies in some fixed compact set. Following [7] we define the $H$-displacement of $\alpha$ as $\operatorname{disp}(\alpha, H)=\left\{\alpha(h) h^{-1}: h \in H\right\}$, and we shall say $\alpha$ has bounded $H$-displacement if $\operatorname{disp}(\alpha, H)$ lies in a fixed compact set. $B(G)$ consists of those $g \in G$ whose conjugacy class has compact closure. Clearly, an inner automorphism $\alpha_{g}$ of $G$ is of bounded displacement if and only if $g \in B(G)$. If $\rho: G \rightarrow G l(W)$ is a continuous linear finite-dimensional representation (not necessarily unitary) we call a continuous function $\phi: G \rightarrow W$ a 1-cocycle (for $\rho$ ) if

$$
\phi\left(g_{1} g_{2}\right)=\rho_{g_{1}}\left(\phi\left(g_{2}\right)\right)+\phi\left(g_{1}\right) \text { for all } g_{1}, g_{2} \in G \text {. }
$$

We now prove

THEOREM 3.1. A connected m.a.p. Lie group $G$ has no nontrivial automorphisms of bounded displacement.

Proof. We first prove the theorem for inner automorphisms, that is, we show $B(G)=Z(G)$. It is clear that $Z(G) \subseteq B(G)$. Consider the case where $G$ is a subgroup of $G l(W)$ and define the linear representation of $G$ on $\operatorname{End}(W)$ by $g \rightarrow \rho_{g}$, where $\rho_{g}(T)=g T g^{-1}$ for $g \in G, T \in \operatorname{End}(W)$. For each $T \in \operatorname{End}(W)$, one gets the (cobounding) continuous 1-cocycle $\phi_{T}: G \rightarrow \operatorname{End}(W)$ defined by $\phi_{T}(g)=T-\rho_{g}(T)$. If $T \in B(G) \subseteq \operatorname{End}(W)$, then $\phi_{T}$ is bounded.

By [18, Proposition 1.6], $\phi_{T} \equiv 0$. That is, $T-\rho_{g}(T)=0$ for every $g \in G$. Hence, $g T g^{-1}=T$ for every $g \in G$, and so $T \subseteq Z(G)$. Thus, $B(G) \subseteq Z(G)$, and the two inclusions imply $B(G)=Z(G)$ in that case.

In general, let $g_{0} \in B(G)$. Then the closure of the $G$-orbit of $g_{0}$ under conjugation, $O_{G}\left(g_{0}\right)^{-}$, is compact and so $\operatorname{Ad}\left(O_{G}\left(g_{0}\right)^{-}\right)$is compact. Because Ad is a representation

$$
\operatorname{Ad}\left(O_{G}\left(g_{0}\right)\right)=O_{\text {Ad } G}\left(\operatorname{Ad} g_{0}\right)
$$

and therefore $\left\{O_{\operatorname{Ad} G}\left(\operatorname{Ad~} g_{0}\right)\right\}^{-}$is compact. Thus $\operatorname{Ad} g_{0} \in B(\operatorname{Ad}(G))$. Since $\operatorname{Ad} G$ is a connected linear m.a.p. group we know by the above $\operatorname{Ad} g_{0} \in Z(\operatorname{Ad} G)$, that is, $\operatorname{Ad}\left(\left[g_{0}, g\right]\right)=1$ for all $g \in G$. This says $\left[g_{0}, g\right] \in \operatorname{ker} A d$, and since $G$ is connected, $\operatorname{ker} \operatorname{Ad}=Z(G)$, and $\left[g_{0}, g\right] \in Z(G)$. Consider the map, $\psi$, from $G$ to $Z(G)$ defined for each $g \in G$ by $\psi(g)=\left[g_{0}, g\right]=\alpha_{g_{0}}(g) g^{-1}$. This map is a continuous homomorphism of $G$ into the abelian group $Z(G)$. But it is well known that a locally compact abelian topological group is MAP, see e.g. [12]. This implies that a m.a.p. group has no nontrivial continuous homomorphisms into a locally compact abelian topological group. Thus, $\psi$ must be trivial. We conclude from this 
that $\left[g_{0}, g\right]=1$ for all $g \in G$ and hence $g_{0} \in Z(G)$. Thus, $B(G)=Z(G)$. This proves Theorem 3.1 completely for inner automorphisms.

To complete the proof of the theorem we require the following result of $\mathbf{J}$. Tits [24, Theorem 3].

If $G$ is a connected Lie group having no nondiscrete normal semisimple compact subgroups, $T^{\prime}$ the largest compact connected subgroup of $Z(G)$, then given any automorphism of bounded displacement, $\alpha$, of $G$, there exists an element $g \in B(G)$ and a homomrphism without fixed points, except $1, \phi: G \rightarrow T^{\prime}$ such that $\alpha$ is defined by the relation $\alpha(x)=g x g^{-1} \phi\left(x^{-1}\right)$.

Continuing the proof, suppose $A$ is a normal semisimple compact subgroup of $G$. If $\pi: G \rightarrow G /[R, R]^{-}$is the canonical projection then $\pi(A)$ is a semisimple compact subgroup of $G /[R, R]^{-}$. There is a conjugate of $\pi(A)$ by an element of $g$, denoted $\pi(A)^{g}$, such that $\pi(A)^{g} \subseteq \pi(S)$, the Levi factor of $G /[R, R]^{-}$(see Theorem 2.2(ii)). Since $\pi(S)$ has no compact factors the group $\pi(A)^{g}$ is trivial. Therefore, also $\pi(A)=(1)$, and $A \subseteq[R, R]^{-}$. But $[R, R]^{-}$is solvable and $A$ is semisimple. Hence, $A$ must be discrete and we can apply the above result of Tits.

If $\alpha$ is an automorphism of bounded displacement of $G$, there exists an element $g \in B(G)$ and a homomorphism $\phi: G \rightarrow T^{\prime}$ without fixed points, except 1 , such that $\alpha(x)=g x g^{-1} \phi\left(x^{-1}\right)$. Since $B(G)=Z(G), g \in Z(G)$ and $\alpha(x)=x \phi\left(x^{-1}\right)$. But the m.a.p. group $G$ has no nontrivial homomorphisms into a compact group, so $\phi$, and therefore also $\alpha$, is trivial.

COROLlARY 3.2. Let $G$ be a m.a.p. connected Lie group and $H$ a closed subgroup of $G$ such that $G / H$ has finite volume. If $\alpha \in \operatorname{Aut}(G)$ leaves $H$ pointwise fixed then $\alpha$ is trivial.

Proof. By [18, Theorem 3.1], $\alpha$ has bounded displacement. Then, by Theorem $3.1, \alpha$ is trivial.

In particular for inner automorphisms we have,

Corollary 3.3 (H. Furstenberg [4, Corollary 3]). If $G$ and $H$ are as in Corollary 3.2, then $Z_{G}(H)=Z(G)$.

Finally we have the following generalization of Corollary 3.2.

THEOREM 3.4. Let $G$ be a m.a.p. connected Lie group and $H$ a closed subgroup of $G$ such that $G / H$ has finite volume. If $\alpha$ is an automorphism of $G$ and $\operatorname{disp}(\alpha, H)$ is bounded, then $\alpha$ is trivial.

Proof. Since $Z(G)$ is a characteristic subgroup of $G, \alpha(Z(G)) \subseteq Z(G)$ and so $\alpha$ induces an automorphism, $\tilde{\alpha}$, of $G / Z(G) \simeq \operatorname{Ad}(G)$. Consider the diagram below.

$$
\begin{array}{ccc}
G & \pi & \operatorname{Ad}(G) \\
\uparrow & & \uparrow
\end{array} \quad \pi: G \rightarrow G / Z(G) \simeq \operatorname{Ad}(G) .
$$

Since $G / H$ has finite volume, $\operatorname{Ad}(G) / \pi(H)^{-}$has volume [7, Corollary 2.10] and $\operatorname{disp}(\alpha, H)$ being bounded implies that $\operatorname{disp}\left(\tilde{\alpha}, \pi(H)^{-}\right)$is bounded. Since $\operatorname{Ad}(G)$ is 
a linear m.a.p. group, $\alpha$ must have bounded displacement [7, Theorem 5.2]. Then, by Theorem 3.1, $\tilde{\alpha}$ is trivial. This means that for every $g \in G, \alpha(g) g^{-1}$ is in $Z(G)$. Define the continuous map $\phi: G \rightarrow Z(G)$ by $\phi(g)=\alpha(g) g^{-1}, g \in G$. Since $\phi$ takes values in $Z(G)$ it is a homomorphism. But $G$ has no nontrivial continuous homomorphisms into an abelian group. Therefore, $\alpha$ is trivial.

4. Extensions of results on minimally almost periodic groups to locally compact connected groups. ${ }^{3}$ Given a locally compact connected group $G$, following $\mathrm{K}$. Iwasawa [14, Theorem 1.5], we let $R$ denote the radical of $G$, i.e. the largest (closed) normal connected solvable subgroup of $G$. In this situation we have the following decomposition theorem due to Y. Matsushima [16, Theorem 1], $G=R \cdot K S$, where $K$ is a compact connected subgroup with trivial radical and $S$ is a semisimple Lie subgroup without compact factors, $[K, S]=(1)$ and $K \cap S$ is a finite group.

As in the case of Lie groups, we let the characteristic subgroup $[R, R]^{-}$, of $G$, denote the closure of the derived of $R$ and we again form the canonical projection $\pi: G \rightarrow G /[R, R]^{-}$. The connected abelian radical, $R /[R, R]^{-}$, of $G /[R, R]^{-}$, can be written as $V \times P$, where $V$ is a vector group and $P$ is a compact group, the $P$ being characteristic in $G /[R, R]^{-}$. Since $P$ is a compact abelian group, the automorphism group of $P, \operatorname{Aut}(P)$, is totally disconnected [14, Corollary p. 14]. Arguing now as in the introduction of $\S 1$, we see that $P$ is centralized by $\pi(K S)$.

LEMMA 4.1. If $G=R \cdot K S$ and $\pi(S)=(1)$ we can replace $V$ in the decomposition above by a vector group of the same dimension, also to be denoted as $V$, which is $\pi(K)$-stable.

Proof. Since $\pi(K S)=\pi(K)$ is a compact subgroup of Aut $(V \times P)$ leaving $P$ fixed, the conclusion follows immediately from [11, Theorem 1.1].

Hereafter, whenever $\pi(K S)=\pi(K)$, we will assume that $V$ is $\pi(K)$-stable.

Definition 4.2. Let $G$ be a locally compact connected group. It is well known that $G=\lim _{\leftarrow} G_{\alpha}$ (projective limit) of connected Lie groups $G_{\alpha}$. If every $G_{\alpha}$ is semisimple we call $G_{\alpha}$ a prosemisimple group.

It has been shown in [17, Corollary 2.5] that the above definition is an intrinsic one, that is, if $G=\lim G_{\alpha}$ is prosemisimple and also $G=\underset{\leftarrow}{\lim } H_{\beta}$, then each $H_{\beta}$ must be a (connected) semisimple Lie group.

Definition 4.3. If $G=R \cdot K S$ is the decomposition above and if $\pi(S)=(1)$, we let $V_{f}$ denote the set of all elements of $V$ which have finite $\pi(K)$-orbit.

As in $\S 1, V_{f}$ is a. normal subgroup of $G /[R, R]^{-}$, and the connectedness of $\pi(K)$ implies that each element of $V_{f}$ is actually $\pi(K)$-fixed. We will show that one may choose a $\pi(K)$-stable complement for $V_{f}$ in $V$, denoted $V_{f}^{\perp}$.

Let $\pi(K)=\lim K_{\alpha}$. Since the radical of $\pi(K)$ is (1), we conclude by [17, Theorem 2.11] that $\pi(K) \overleftarrow{\text { is }}$ prosemisimple and therefore each $K_{\alpha}$ is a semisimple Lie group. Let $\rho: \pi(K) \rightarrow G l(V)$ be the continuous homomorphism induced by the action of $\pi(K)$ on $R /[R, R]^{-}$restricted to $V$. By [17, Lemma 2.2] there exists an $\alpha$ and a

\footnotetext{
${ }^{3}$ Unfortunately in this section we do not calculate $n(G)$ as in $\$ 1$. Our results in $\$ 4$ will be pursued further.
} 
compact subgroup $C_{\alpha}$ such that $K_{\alpha}=\pi(K) / C_{\alpha}$ and $\rho\left(C_{\alpha}\right)=(1)$, i.e., $\rho$ induces a continuous homomorphism $\tilde{\rho}$ of $K_{\alpha}$ such that the following diagram commutes.

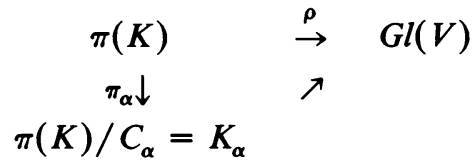

Thus, we may consider $\rho$ as a (unitary) representation of the compact group $K_{\alpha}$ on $V$. Since $V_{f}$ is $\pi(K)$-stable, it is therefore $K_{\alpha}$-stable, and now Weyl's theorem enables us to find a $K_{\alpha}$-stable, and therefore $\pi(K)$-stable, complement in $V$ written as $V_{f}^{\perp}$, which as before is normal in $G /[R, R]^{-}$.

COROLlARY 4.4. If $G=\lim G_{\alpha}$ is a locally compact connected group, then $G$ is m.a.p. if and only if each $G_{\alpha} \stackrel{\leftarrow}{\text { is }}$ m.a.p.

Proof. Suppose that each $G_{\alpha}$ is m.a.p. and let $\rho$ be an irreducible representation of $G$. By [17, Lemma 2.2], for some $\alpha, \rho$ induces a representation $\tilde{\rho}$, of $G_{\alpha}$, such that $\tilde{\rho} \circ \pi_{\alpha}=\rho$. Since $G_{\alpha}$ is m.a.p., $\tilde{\rho}$ is trivial and hence $\rho$ is trivial. Therefore, $G$ is m.a.p. The converse is clear.

LEMMA 4.5. If $G$ is a locally compact connected group with decomposition $G=R$. $K S$, then both $S$ and $[R, R]^{-}$are contained in $n(G)$. If $R$ is abelian, and $S=(1)$ then $V_{f}^{\perp}$ is also contained in $n(G)$.

Proof. Let $G=\lim G_{\alpha}$ with $\pi_{\alpha}: G \rightarrow G_{\alpha}$ the corresponding projections, and let $\rho$ be an irreducible representation of $G$. Then $\rho$ induces a representation $\tilde{\rho}$ of some $G_{\alpha}$. We have $\rho([R, R])=\tilde{\rho}\left(\pi_{\alpha}([R, R])\right) \subseteq \tilde{\rho}\left(\left[R_{\alpha}, R_{\alpha}\right]\right)=(1)$ (by Lemma 1.3). Therefore, $\rho([R, R])=(1)$, and by continuity $\rho\left([R, R]^{-}\right)=(1)$. Hence, $[R, R]^{-} \subseteq n(G)$. The remainder of Lemma 4.5 is proven exactly as in Lemma 1.3. This completes the proof.

LEMMA 4.6. If $G$ is a locally compact connected group, then $G$ is m.a.p. if and only if $G /[R, R]^{-}$is m.a.p.

Proof. This is proven exactly as in the Lie group case by first showing $n(G)=\pi^{-1}\left(n\left(G /[R, R]^{-}\right)\right)$(see proof of Theorem 1.1) and then applying the argument of Lemma 2.1 .

The next theorem generalizes Theorem 2.2 to locally compact connected groups.

THEOREM 4.7. Let $G$ be a locally compact connected group. Then the following are equivalent.

(i) $G$ is m.a.p.

(ii) $G /[R, R]^{-}$can be written as $V \cdot \pi(S)$ with all nonzero elements of $V$ of unbounded $\pi(S)$-orbit. (In particular, $G /[R, R]^{-}$is a (m.a.p.) Lie group.)

(iii) $G / R$ is a semisimple Lie group without compact factors and every nonzero element of $R /[R, R]^{-}$is of unbounded $\pi(S)$-orbit.

(iv) $G / R$ is a semisimple Lie group without compact factors and $[R, R]^{-}=\{r \in$ $\left.R:[G, r] \subseteq[R, R]^{-}\right\}$.

(v) $G / R$ is a semisimple Lie group without compact factors and $G=[G, G]^{-}$. 
Proof. Let $G / R=\lim _{\leftarrow} H_{\alpha}$ with corresponding projections $\pi_{\alpha}: G / R \rightarrow H_{\alpha^{*}}$ (i) $\rightarrow$ (ii). Suppose $G$ is m.a.p. Then, $G / R$ is m.a.p. and therefore each $H_{\alpha}$ is also m.a.p. (Corollary 4.4). Thus, every connected Lie group $H_{\alpha}$ is m.a.p. and semisimple, and therefore has no compact factors (Lemma 1.5(b)). Let $M$ be a compact connected normal subgroup of $G / R$. For each $\alpha, \pi_{\alpha}(M)$ is a compact connected normal subgroup of $H_{\alpha}$ and so $\pi_{\alpha}(M)=(1)$ and we have $M \subseteq \cap_{\alpha} \operatorname{ker} \pi_{\alpha}=(1)$. Hence, $M=(1)$ and so $G / R$ has no nontrivial compact connected normal subgroups. Therefore, $K=(1)$ and $G=R \cdot S$. This implies that $G / R$ is a semisimple Lie group without compact factors. The group $G /[R, R]^{-}$is m.a.p. since $G$ is m.a.p., and we have written its connected abelian radical $R /[R, R]^{-}$as $V \times P$. We have seen that the compact connected group $P$ is fixed under the action of $\pi(K S)$. Since $P$ is also abelian and commutes with $V$ it must be contained in $Z\left(G /[R, R]^{-}\right)$, the identity component of the center of $G /[R, R]^{-}$. We see that $G /[R, R]^{-}$ $=\lim _{\leftarrow} G_{\alpha} /\left[R_{\alpha}, R_{\alpha}\right]^{-}\left(G=\lim G_{\alpha}\right.$ and for each $\alpha, \phi_{\alpha}: G /[R, R]^{-} \rightarrow G_{\alpha} /\left[R_{\alpha}, R_{\alpha}\right]^{-}$ will $\overleftarrow{\leftarrow}$ be the corresponding projection), and each $G_{\alpha} /\left[R_{\alpha}, R_{\alpha}\right]^{-}$is a connected m.a.p. Lie group (Lemma 4.1), with abelian radical $R_{\alpha} /\left[R_{\alpha}, R_{\alpha}\right]^{-}$. Hence by Theorem 1.2, for each $\alpha$,

$$
G_{\alpha} /\left[R_{\alpha}, R_{\alpha}\right]^{-}=n\left(G_{\alpha} /\left[R_{\alpha}, R_{\alpha}\right]^{-}\right)=\left(V_{\alpha}\right)_{f}^{\perp} \tilde{\pi}_{\alpha}\left(S_{\alpha}\right)
$$

where $G_{\alpha} /\left[R_{\alpha}, R_{\alpha}\right]^{-}=R_{\alpha} /\left[R_{\alpha}, R_{\alpha}\right]^{-} \cdot \tilde{\pi}_{\alpha}\left(S_{\alpha}\right)$ is the Levi decomposition of $G_{\alpha} /\left[R_{\alpha}, R_{\alpha}\right]^{-}\left(\tilde{\pi}_{\alpha}: G_{\alpha} \rightarrow G_{\alpha} /\left[R_{\alpha}, R_{\alpha}\right]^{-}\right.$is the canonical projection), and $R_{\alpha} /\left[R_{\alpha}, R_{\alpha}\right]^{-}=V_{\alpha} \times T_{\alpha}, V_{\alpha}$ a vector group, and $T_{\alpha}$ a toroidal group. From (26), $\left(V_{\alpha}\right)_{f}=(1)$ and since for each $\alpha, Z\left(G_{\alpha} /\left[R_{\alpha}, R_{\alpha}\right]^{-}\right)_{0} \subseteq\left(V_{\alpha}\right)_{f}=(1)$ and $\phi_{\alpha}\left(Z\left(G /[R, R]^{-}\right)_{0}\right) \subseteq Z\left(G_{\alpha} /\left[R_{\alpha}, R_{\alpha}\right]^{-}\right)_{0}$ we have $Z\left(G /[R, R]^{-}\right)_{0} \subseteq \cap \operatorname{ker} \phi_{\alpha}=$ (1). Thus $P=(1)$, and $R /[R, R]^{-}=V$ is a vector group. Thus, $G /[R, R]^{-}=V$. $\pi(S)$ is a Lie group and it is m.a.p. since $G$ is m.a.p. We apply the argument of Theorem 2.2 (i $\rightarrow$ ii) to complete the proof of (ii).

Conversely, assume (ii) holds. Then $G /[R, R]^{-}$is a Lie group and by Theorem 2.2 (ii $\rightarrow \mathrm{i}$ ), it is m.a.p. By Lemma $4.6, g$ is also m.a.p.

(i $\rightarrow$ iii). Assume that (i) holds. By (ii) above, $G / R$ is a semisimple Lie group without compact factors. The remainder of (iii) is shown as in Theorem $2.2(\mathrm{i} \rightarrow \mathrm{iii})$. Conversely, if (iii) holds, then $K=(1)$, and $P=(1)$ since $P$ is $\pi(K S)$-fixed. Therefore, $G /[R, R]^{-}=V \cdot \pi(S)$ with all nonzero elements of $V$ of unbounded $\pi(S)$-orbit. By (ii), $G$ is m.a.p.

(i $\rightarrow$ iv). Assume that $G$ is m.a.p. From (iii), $G / R$ is a semisimple Lie group without compact factors. Exactly as in Theorem $2.2(\mathrm{i} \rightarrow \mathrm{iv})$ we show that $[R, R]^{-}$ $=\left\{r \in R:[G, r] \subseteq[R, R]^{-}\right\}$. Conversely, if (iv) holds, we apply the argument of Theorem 2.2 (iv $\rightarrow$ i).

$(\mathrm{i} \rightarrow \mathrm{v}$ ). If (i) holds, by (iv), $G / R$ is a semisimple Lie group without compact factors. To see that $G=[G, G]^{-}$, argue as in Theorem $2.2(\mathrm{i} \rightarrow \mathrm{v})$. In the other direction, see Theorem $2.2(\mathrm{v} \rightarrow \mathrm{i})$.

This completes the proof of Theorem 4.7.

We conclude this paper with an alternate, and quite simple proof of the following well-known result. 
Corollary 4.8 (Freudenthal-Weil [3, p. 129]). A locally compact connected group $G$ is MAP if and only if $G$ is the direct product of a vector group and a compact group.

Proof. Let $G$ be MAP with decomposition $G=R \cdot K S$. Here, $n(G)=(1)$, and by Lemma 4.5 both $S$ and $[R, R]^{-}$are contained in $n(G)$, whence $[R, R]^{-}=S=$ (1). Then, $R$ is abelian and so by Lemma 4.5, $V_{f}^{\perp} \subseteq n(G)$. Thus, $V_{f}^{\perp}=(1)$, $V=V_{f}$, and $G=(V \times P) \cdot K$. Because $G=V \cdot P K$ and both $V$ and $P K$ are closed subgroups and $V \cap P K=(1)$ with $P K$ central in $G$, therefore $G=V \times$ $P K$. Thus, $G$ is the direct product of a vector group and a compact group.

Conversely, let $G=V \times M$, with $V$ a vector group and $M$ a compact group. By Proposition 1.6, $n(G)=n(V) \times n(M)$ and since both $V$ and $M$ are MAP, $n(G)=$ (1).

\section{BIBLIOGRAPHY}

1. Frank J. Adams, Lectures on Lie groups, Benjamin, New York, 1969.

2. A. H. Clifford, Representations induced in an invariant subgroup, Ann. of Math. 38 (1937), 533-550.

3. H. Freudenthal, Topologische Gruppen mit genugend vielen fastperiodischen Funktionen, Ann. of Math. 37 (1936), 57-77.

4. H. Furstenberg, A note on Borel's density theorem, Proc. Amer. Math. Soc. 55 (1976), 209-212.

5. M. Gotô, Faithful representations of Lie groups. I, Math. Japonicae 1 (1948), 107-119.

6. __ Linear representations of topological groups, Proc. Amer. Math. Soc. 1 (1950), 425-439.

7. F. P. Greenleaf and M. Moskowitz, Groups of automorphisms of Lie groups: density properties, bounded orbits, and homogeneous spaces of finite volume, Pacific J. Math. (to appear).

8. F. P. Greenleaf, M. Moskowitz and L. P. Rothschild, Unbounded conjugacy classes in Lie groups and location of central measures, Acta Math. 132 (1974), 225-243.

9. Automorphisms, orbits, and homogeneous spaces of nonconnected Lie groups, Math. Ann. $212(1974 / 75), 145-155$.

10. S. Grosser and M. Moskowitz, On central topological groups, Trans. Amer. Math. Soc. 127 (1967), 317-340.

11. $1-40$. , Compactness conditions in topological groups. I, II, J. Reine Angew. Math. 246 (1971),

12. G. Hochschild, The structure of Lie groups, Holden-Day, San Francisco, Calif., 1965.

13. T. J. Huang, On topologies of maximally almost periodic groups, Proc. Amer. Math. Soc. 69 (1978), 251-254.

14. K. Iwasawa, On some types of topological groups, Ann. of Math. 50 (1949), 507-558.

15. M. Kuranishi, On non-connected maximally almost periodic groups, Tôhoku Math. J. 2 (1950), 40-46.

16. Y. Matsushima, On the decomposition of an (L)-group, J. Math. Soc. Japan 1 (1950), 264-274.

17. M. Moskowitz, On proreductive groups, Proc. Cambridge Philos. Soc. 76 (1974), 401-406.

18. Some remarks on automorphisms of bounded displacement and bounded cocycles, Monatsh.

Math. 85 (1978), 323-336.

19. __ On the density theorems of Borel and Furstenberg, Ark. Mat. 16 (1978), 11-27.

20. S. Murakami, Remarks on the structure of maximally almost periodic groups, Osaka J. Math. 1 (1950), 119-129.

21. J. von Neumann, Almost periodic functions in a group, Trans. Amer. Math. Soc. 36 (1934), 445-492.

22. J. von Neumann and E. P. Wigner, Minimally almost periodic groups, Ann. of Math. 41 (1940), 746-750.

23. M. S. Raghunathan, Discrete subgroups of Lie groups, Springer-Verlag, Berlin and New York, 1972.

24. J. Tits, Automorphisms a déplacement borné des groupes de Lie, Topology 3 (1964), 97-107. 
25. B. L. van der Waerden, Stetigkeitssatzeder halbeinfachen LieschenGruppen, Math. Z. 36 (1933), 780-786.

26. A. Weil, L'integration dans les groupes topologiques et ses applications (2nd ed.), Hermann, Paris, 1965.

Department of Mathematics, Adelphi University, SOUth Street, Garden Ctry, New York 11530

(CUNY), Graduate School and University Centre, 33 West 42ND Strebt, New York, New YoRk 10036

Current address: Department of Mathematics, C. W. Post College, Greenvale, New York 11548 